\title{
SPECTRAL PROPERTIES OF COMPACT LATTICE HOMOMORPHISMS
}

\author{
BY
}

\author{
ANTHONY W. WICKSTEAD
}

\begin{abstract}
Given two nonzero eigenvalues of a lattice homomorphism on a relatively uniformly complete vector lattice, of different moduli and with at least one isolated in the set of all eigenvalues, we show that corresponding eigenvectors must be disjoint. The analogous result for the approximate point spectrum of a lattice homomorphism on a Banach lattice is deduced. We give an infinite spectral decomposition for a lattice homomorphism, on a Banach lattice with order continuous norm, which is compact and has an adjoint which is also a lattice homomorphism. From this we deduce that if it has nonnegative spectrum, then it is the direct sum of a nilpotent lattice homomorphism and one that is central.
\end{abstract}

1. Introduction. Much work has been done on the spectra of lattice homomorphisms on Banach lattices $([7,13$, and 14] for example). There are also well known nice properties of compact operators on an arbitrary Banach space. It is to be expected that compact lattice homomorphisms will have particularly well-behaved spectra. This indeed turns out to be the case. Although the present work was prompted by the recent complete description of compact lattice homomorphisms of Banach lattices (see [4] or [12]), the results do not make use of that description, so the paper is relatively self-contained.

$\$ 2$ contains a result on the location of eigenvectors of lattice homomorphisms. It is valid without the assumption of compactness, but is especially significant in that case. $\$ 3$ investigates how close to being central a compact lattice homomorphism with nonnegative spectrum must be.

We will work throughout with complex Banach lattices and the corresponding classes of complex linear operators on them. An account of the complexification of a real vector lattice, and of the linear operators on it, may be found in [5, Chapter 2, §11]. We will adopt that book as our source of terminology and notation.

2. Disjointness of eigenvectors. The classical result that eigenvectors of a Hermitian operator on a Hilbert space, which correspond to distinct eigenvalues, must be disjoint is so far fairly isolated in the mathematical literature. The simplest lattice homomorphisms to study, namely those of the form $f \mapsto h \cdot f$ on $C(X)$, are easily seen to share this property (using, of course, an order theoretic definition of disjointness). On the other hand, even the relatively simple operator $T$ on $C([-1,1])$, defined by $T f(x)=f(-x)$, does not. If $g(x)=1$ and $h(x)=x$, then $T g=1 \cdot g$ and $T h=(-1) h$, but $g$ and $h$ are not disjoint. Nevertheless, there does seem to be some

Received by the editors February 26, 1981 and, in revised form, June 23, 1981.

1980 Mathematics Subject Classification. Primary 47B05, 47B55.

(C) 1982 American Mathematical Society 0002-9939/81/0000-1019/\$02.75 
general result lurking here. First let us recall that a vector lattice $E$ is relatively uniformly complete if, for every $x \in E$, the ideal generated by $|x|$ is complete for the order unit norm induced by $|x|$. This is the same as Axiom (OS) of [5, Chapter 2, Definition 1.8]. $\operatorname{P\sigma }(T)$ will denote the set of all eigenvalues of the linear operator $T$. If $K$ is any subset of $\mathbf{C}$ then $K^{\sim}$ will denote its isolated points.

THEOREM 1. If $T$ is a lattice homomorphism on a relatively uniformly complete vector lattice $E, \lambda \in \operatorname{Po}(T) \backslash\{0\}, \mu \in P \sigma(T) \backslash\{0\}, T x=\lambda x, T y=\mu y$ and $|\lambda| \neq|\mu|$, then $|x| \wedge|y|=0$.

Proof. If $|z| \leqslant p(|x|+|y|)$, then

$$
\begin{aligned}
|T z| & =T|z| \leqslant p(T|x|+T|y|)=p(|T x|+|T y|) \\
& \leqslant p(|\lambda x|+|\mu y|) \leqslant p \cdot \max \{|\lambda|,|\mu|\}(|x|+|y|) .
\end{aligned}
$$

Thus we can restrict our attention to the ideal generated in $E$ by $|x|+|y|$. By Kakutani's representation theorem for abstract $M$-spaces, this ideal is linearly order isomorphic to some $C(\Omega)$, where $\Omega$ is a compact Hausdorff space (see [5, Chapter 2, Theorem 7.4]).

Suppose, therefore, that $E$ is such a space $C(\Omega)$ and that $x, y \in C(\Omega) . T$ is of the form

$$
T f(\omega)=H(\omega) f(\phi \omega),
$$

where $h \in C(\Omega)_{+}$and $\phi: \Omega \rightarrow \Omega$ is continuous on $\Omega \backslash h^{-1}(0)$ [13, Proposition 1.1]. Let $0<\alpha<1$ and consider the function on $\Omega$ defined by

$$
\begin{aligned}
z_{\alpha}(\omega) & =x(\omega)|y(\omega)|^{\alpha} /|x(\omega)|^{\alpha} \quad(x(\omega) \neq 0), \\
& =0 \quad(x(\omega)=0) .
\end{aligned}
$$

$z_{\alpha} \in C(\Omega)$ and is identically zero only if $x \cdot y$ is identically zero, i.e. only if $|x| \wedge|y|=0$, which is what we wish to establish. We therefore assume that each $z_{\alpha} \neq 0$ and obtain a contradiction.

We know that

$$
h(\omega) x(\phi \omega)=\lambda x(\omega)
$$

and that

$$
h(\omega) y(\phi \omega)=\mu y(\omega)
$$

for each $\omega \in \Omega$. Hence, if $|x(\phi \omega)| \neq 0$ and $h(\omega) \neq 0$, then

$$
\begin{aligned}
T z_{\alpha}(\omega) & =h(\omega) z_{\alpha}(\phi \omega)=h(\omega) x(\phi \omega)|y(\phi \omega)|^{\alpha} /|x(\phi \omega)|^{\alpha} \\
& =\lambda x(\omega)|\mu y(\omega) / \lambda x(\omega)|^{\alpha}=\lambda|\mu / \lambda|^{\alpha} z_{\alpha}(\omega) .
\end{aligned}
$$

If $h(\omega)=0$ or $x(\phi \omega)=0$ then $T z_{\alpha}(\omega)=0$ and $x(\omega)=0$ by (1), so that $z_{\alpha}(\omega)=0$.

Thus $\lambda \cdot|\mu / \lambda|^{\alpha} \in \operatorname{P\sigma }(T)$ for each $0<\alpha<1$. Provided $\mu, \lambda \neq 0$ and $|\lambda| \neq|\mu|$, this contradicts $\lambda$ being isolated in $\operatorname{P\sigma }(T)$ and completes the proof.

It will be clear from the proof that the relative uniform completeness of $t$ could be weakened to the assumption that the image of each principal ideal under the Kakutani representation is an algebra. The condition cannot, however, just be dropped. Nor can any of the others, as the following examples show. 
EXAMPLE (1). Let $E$ be the vector lattice $P A[0,1]$ of piecewise affine functions on $[0,1]$. Thus for each element $f$ of $E,[0,1]$ can be divided into a finite number of subintervals on each of which $f$ is affine. Define a function $\phi:[0,1] \rightarrow[0,1]$ by specifying that $\phi(\omega)=\omega / 2$. The linear operator $T$ defined by $T f(\omega)=f(\phi \omega)$ is a lattice homomorphism of $E$ into itself. If $f$ is an eigenvector of $T$ corresponding to the eigenvalue $\lambda$, then suppose $f$ has slope $m$, on some subinterval $I$, of $\left[2^{-(n+1)}, 2^{-n}\right]$ $(n>0)$. On $\phi^{-1}(I), T f$ will have slope $m / 2$. As $T f=\lambda f, f$ will have slope $m / 2 \lambda$ on $\phi^{-1}(I)$. Thus if $f$ somewhere has slope $m$, it will also have slopes $(2 \lambda)^{k} m$ (for each $k \in N)$ on some subinterval of $[0,1]$. This will contradict $f$ being in $P A[0,1]$, as $f$ would have infinitely many different slopes, unless $\lambda=\frac{1}{2}$, or zero is the only value of $m$. The second case implies that $f$ is constant, and therefore that $\lambda=1$. It follows that $\operatorname{Po}(T)=\left\{\frac{1}{2}, 1\right\}$ as $T g=\frac{1}{2} g$ where $g(\omega)=\omega$, and $T h=h$ where $h(\omega)=1$. All the hypotheses of Theorem 1 are satisfied except the relative uniform completeness of $E$, yet the conclusion clearly fails.

EXAmple (2). Define $T$ on $\mathbf{C}^{2}$ by $T(\alpha, \beta)=(\alpha, \alpha)$. Then $T$ is a lattice homomorphism with eigenvalues 0 and 1 . Corresponding eigenvectors are $(0,1)$ and $(1,1)$ which are not disjoint. All the conditions of Theorem 1 are satisfied except that one of the eigenvalues is zero.

Example (3). Take $E$ to be $c_{0}$ and define $T$ on $E$ by

$$
(T z)_{n}=z_{n+1} \text {. }
$$

Then $\operatorname{P\sigma }(T)=\{\lambda \in \mathrm{C}:|\lambda|<1\}$ and the eigenvector corresponding to $\lambda$ is $\left(\lambda^{n}\right)$. Taking $\lambda, \mu \in P \sigma(T) \backslash\{0\}$ with distinct moduli shows that the conclusion of Theorem 1 fails, although all the conditions are satisfied except that the eigenvalues are isolated.

Theorem 1 has particular relevance to compact lattice homomorphisms as all nonzero points of the spectrum are then isolated eigenvalues.

For lattice homomorphisms on a Banach lattice we can use Theorem 1 and a technique used in Lotz [2] to prove a related result. Recall that the complex number $\lambda$ is an approximate eigenvalue of the linear operator $T$ on a Banach space $E$ if there is a sequence $\left(x_{n}\right)$ in $E$ with $\left\|x_{n}\right\|=1$ and $\left\|(T-\lambda) x_{n}\right\| \rightarrow 0$. The set of all approximate eigenvalues of $T$ is denoted by $A \sigma(T)$.

If $E$ is a Banach space, $l_{\infty}(E)$ will denote the space of all bounded sequences in $E$, normed by $\left\|\left(x_{n}\right)\right\|=\sup \left\{\left\|x_{n}\right\|: n \in N\right\}$, and $c_{0}(E)$ the closed subspace of nullsequences in $E$. Let $\mathscr{P}(E)$ denote the quotient space $l_{\infty}(E) / c_{0}(E)$. If $T$ is a bounded linear operator on $E$ and $\left(x_{n}\right) \in l_{\infty}(E)$ (resp. $c_{0}(E)$ ), then $\left(T x_{n}\right) \in l_{\infty}(E)$ (resp. $\left.c_{0}(E)\right)$. We may thus define $\mathscr{P}(T)$ on $\mathscr{P}(E)$ by

$$
\mathcal{P}(T)\left(\left(x_{n}\right)+c_{0}(E)\right)=\left(T x_{n}\right)+c_{0}(E),
$$

to obtain a bounded linear operator on $\mathscr{P}(E)$. This construction is of use as [2, Theorem 2.4], $\operatorname{P\sigma }(\mathscr{P}(T))=A \sigma(\mathscr{P}(T))=A \sigma(T)$. If $E$ is a Banach lattice and we extend the order to $l_{\infty}(E)$ term by term, then give $\mathscr{P}(E)$ the quotient order, then $\mathcal{P}(E)$ is also a Banach lattice [2, Theorem 2.5]. If $T$ is a lattice homomorphism on $E$, then $\mathscr{P}(T)$ is a lattice homomorphism on $\mathscr{P}(E)$ [2, Theorem 2.6]. 
COROllary 2. Let $E$ be a Banach lattice and $T$ a lattice homomorphism on $E$. If $\lambda \in A \sigma(T) \sim\{0\}, \mu \in A \sigma(T) \backslash\{0\},\left(x_{n}\right)$ and $\left(y_{n}\right)$ are sequences of unit vectors in $E$ such that $\left\|(T-\lambda) x_{n}\right\| \rightarrow 0$ and $\left\|(T-\mu) y_{n}\right\| \rightarrow 0$ and $|\lambda| \neq|\mu|$, then $\left\|\left|x_{n}\right| \wedge\left|y_{n}\right|\right\|$ $\rightarrow 0$.

Proof. $\lambda, \mu \in P \sigma(\mathcal{P}(T)) \tilde{i}\{0\}$ and

$$
\begin{aligned}
& \mathcal{P}(T)\left(\left(x_{n}\right)+c_{0}(E)\right)=\lambda\left(\left(x_{n}\right)+c_{0}(E)\right), \\
& \mathcal{P}(T)\left(\left(y_{n}\right)+c_{0}(E)\right)=\mu\left(\left(x_{n}\right)+c_{0}(E)\right) .
\end{aligned}
$$

By applying Theorem 1 to the operator $\mathscr{P}(T)$ on $\mathscr{P}(E)$, we see that $\left(x_{n}\right)+c_{0}(E)$ and $\left(y_{n}\right)+c_{0}(E)$ are disjoint, i.e. $\left|x_{n}\right| \wedge\left|y_{n}\right| \in c_{0}(E)$, which is the desired conclusion.

It should be noted that, since $A \sigma(T)$ contains the boundary of $\sigma(T)$, the spectrum of $T, A \sigma(T) \tilde{)} \supset \sigma(T)$.

3. Compact central lattice homomorphisms. A linear operator $T$ on a vector lattice $E$ is central if there exists a real $p \geqslant 0$ such that $|T x| \leqslant p|x|$ for all $x \in E$. These have been studied in detail. A positive central operator is a lattice homomorphism and has nonnegative spectrum. One known partial converse is that an invertible lattice homomorphism with positive spectrum must be central [6, Theorem 2.1]. Once noninvertible operators are considered, such a result becomes impossible as there are quasi-nilpotent noncentral lattice homomorphisms. The compact central operators on a Banach lattice have been described in [10, Theorem 4.1] and [4, Theorem 7.2]. When dealing with Banach lattices with order continuous norms, i.e. those for which every net decreasing to 0 converges to 0 in norm, it is possible to describe fairly completely those compact lattice homomorphisms with nonnegative spectrum, provided we assume that the adjoint operator is also a lattice homomorphism. Although this condition may seem rather artificial, it is one that is satisfied by central lattice homomorphisms, has already played a role in other results on the spectral properties of lattice homomorphisms, and cannot be omitted as we show by a simple example.

THEOREM 3. Let $E$ be a Banach lattice with an order continuous norm and let $T$ be a compact lattice homomorphism on $E$ whose adjoint, $T^{*}$, is also a lattice homomorphism. $E$ is a (possibly infinite) order direct sum $\left(\oplus_{r} H_{r}\right) \oplus K$, where $r$ ranges over positive reals in $\sigma(T)$ where each $H_{r}$ and $K$ are $T$-invariant ideals, $\left.T\right|_{K}$ is quasi-nilpotent, each $H_{r}$ is finite dimensional and $\sigma\left(\left.T\right|_{H_{r}}\right)=\{\lambda \in \sigma(T):|\lambda|=r\}$.

Proof. Let

$$
I=\bigcap_{n=1}^{\infty} \overline{T^{n}(E)} \text { and } J=\left[\bigcup_{n=1}^{\infty}\left(T^{n} E\right)^{\perp}\right]^{-} \text {. }
$$

If $S$ is a compact lattice homomorphism and $S^{*}$ is also a lattice homomorphism, then for each $x \geqslant 0, S([0, x])$ is dense in $[0, S x]$ by [3, Proposition 1.2] $([0, x]$ denotes the order interval $\{y: 0 \leqslant y \leqslant x\})$. But $S([0, x])$ is compact, so $S([0, x])=$ $[0, S x]$, so $S E$ is actually an ideal in $E$. Hence each $T^{n} E$ is an ideal in $E$, so is its 
closure and hence their intersection $I$. The sets $\left(T^{n} E\right)^{\perp}$ form an increasing set of closed ideals, so is an ideal and hence also is $J$.

Each $\left(T^{n} E\right)^{\perp}$ is orthogonal to $I$, so we see that $I \perp J$. If $x \in E$ then we can write $x=y_{n}+z_{n}$, where $y_{n} \in \overline{T^{n} E}$ and $z_{n} \in\left(T^{n} E\right)^{\perp}$; as in a Banach lattice with an order continuous norm, every closed ideal is a projection band [5, Chapter II, Theorem 5.10]. This decomposition is unique, and if $n>m$ then $T^{n} E \subset T^{m} E$ (so that $\left.\left(T^{n} E\right)^{\perp} \supset\left(T^{m} E\right)^{\perp}\right)$, so that $0 \leqslant y_{n} \leqslant y_{m}$ and $x \geqslant z_{n} \geqslant z_{m}$. As $E$ has an order continuous norm $y_{n} \rightarrow y \in E$ and $z_{n} \rightarrow z \in E$, and clearly $x=y+z$. As $\overline{T^{n} E}$ is an ideal in $E$ and $0 \leqslant y \leqslant y_{n} \in \overline{T^{n} E}, y \in \overline{T^{n} E}$. Thus $y \in I$. Also note that each $z_{n} \in J$, which is closed, so $z \in J$. Thus $I$ and $J$ are complementary bands.

If $x \in \overline{T^{n} E}$, then $x=\lim _{k \rightarrow \infty} T^{n} x_{k}$ for some $x_{k} \in E$, so $T x=\lim _{k \rightarrow \infty} T^{n+1} x_{k} \in \overline{T^{(n+1)}} E$. Thus if $x \in I$, then $x \in \cap_{n=2}^{\infty} T^{n} E \subset I$. Also note that $x \perp T^{n} E$ implies that $T x \perp T^{n+1} E$, so $\cup\left(T^{n} E\right)^{\perp}$ is $T$-invariant and by continuity so is $J$.

$\left.T\right|_{J}$ is compact, so if $\sigma\left(\left.T\right|_{J}\right) \neq\{0\}$, there is a nonzero eigenvalue $\lambda$ of $T$ and a corresponding eigenvector $j \in J$. But then $j=\left(T^{n} j\right) / \lambda^{n} \in T^{n} E$ for all $n, j \in I$. This implies that $j=0$, so $\left.T\right|_{J}$ is quasi-nilpotent.

If $P$ denotes the band projection onto $I$, then we have established that $P T=T P$ as both $I$ and $J$ are $T$-invariant. Each $x \in I$ is in $\overline{T E}$, so there is a sequence $x_{k}$ in $E$ with $T x_{k} \rightarrow x$, and hence $P T x_{k} \rightarrow P x=x$. Thus $T\left(P x_{k}\right) \rightarrow x$, and $P x_{k} \in I$, so $T I$ is dense in $I$. In $T I$ every order interval is compact, so it follows by [8, Theorem 1], [9, Theorem 5], or [4, Theorem 4.9] that $I$ may be identified with an ideal in $l_{\infty}$ which contains each $e_{n}$ ( $n$th coordinate 1 , the remainder 0 ) corresponding to an element of $I$ of unit norm. We will now make such an identification for $I$.

$\left.T\right|_{I}$ may be identified with the operator $\left(x_{n}\right) \rightarrow\left(\alpha_{n} x_{\phi n}\right)$ where $\phi: \mathbf{N} \rightarrow \mathbf{N}$ and $\alpha_{n}$ is a sequence of nonnegative reals, because $T$ is a lattice homomorphism. Since $\left(\left.T\right|_{I}\right)^{*}$ is a lattice homomorphism $\phi$ must be $1-1$, and since $T$ is compact $\alpha_{n} \rightarrow 0$. Note also that the definition of $I$ implies that each $\alpha_{n}>0$.

Let $i$ be an eigenvector of $T$ in $I$ corresponding to a nonzero eigenvalue $\lambda$. If $A=\left\{n \in N: i_{n} \neq 0\right\}$ then clearly $\phi(A) \subset A$. If $n \in A$ then the sequence $n, \phi n$, $\phi^{2} n, \ldots$ can take only finitely many values, else $\phi^{k} n \rightarrow \infty$ as $k \rightarrow \infty$ and hence $\alpha_{\phi^{k} n} \rightarrow 0$ as $k \rightarrow \infty$. But then $\left(T_{i}\right)_{n}=\alpha_{n} i_{\phi n}=\lambda i_{n}$ implies that

$$
i_{\phi^{k} n}=i_{n} \cdot \lambda^{k} / \alpha_{n} \alpha_{\phi n} \ldots \alpha_{\phi^{k} n} \rightarrow \infty,
$$

contradicting $\left(i_{n}\right) \in l_{\infty}$. Thus for some $N, \phi^{N} n=n$. Clearly $\left\{n, \phi n, \ldots, \phi^{N-1} n\right\}$ is $\phi$-invariant and as $\phi$ is $1-1$ so is its complement in $A$. It follows that $i^{\prime}$ defined by

$$
\begin{aligned}
i_{k}^{\prime} & =i_{k}, \quad \text { if } k \in\left\{n, \phi n, \ldots, \phi^{N-1} n\right\}, \\
& =0, \quad \text { otherwise, }
\end{aligned}
$$

is an eigenvector of $T$ corresponding to $\lambda$. If $A$ were infinite we could obtain infinitely many orthogonal eigenvectors of $T$ corresponding to $\lambda$, which would contradict the compactness of $T$.

It follows that $A$ is finite and that $\left.\phi\right|_{A}$ is a bijection. Hence for each eigenvector $i$ of $T$ in $I$ which corresponds to a nonzero eigenvalue, the ideal generated by $i$ is finite dimensional and $T$ restricted to it is invertible. 
By Theorem 1 the ideals corresponding to eigenvalues of distinct modulus must be orthogonal. We thus have that each ideal $H_{r}$ generated by the set $\{i \in I:|T i|=r|i|\}$, for positive $r$ in $\sigma(T)$, is a finite-dimensional ideal in $I$, and that $\sigma\left(\left.T\right|_{H_{r}}\right)=\sigma(T) \cap$ $\{\lambda \in \mathbf{C}:|\lambda|=r\}$. The order continuity of the norm in $E$ guarantees that $\left(\sum H_{r}\right)^{-}$is a projection band in $I$ (and hence in $E$ ) which is $T$-invariant. Clearly the compact operator $\left.T\right|_{\left(\Sigma H_{r}\right)^{\perp}}$ can have no nonzero eigenvalues, so is quasi-nilpotent. Taking $K=J \oplus\left(\Sigma H_{r}\right)^{\perp}$ gives the decomposition of the statement of the theorem.

COROLlaRY 4. Let $E$ be a Banach lattice with an order continuous norm and let $T$ be a compact lattice homomorphism on $E$ whose adjoint $T^{*}$ is also a lattice homomorphism. If $\sigma(T) \subseteq \mathbf{R}_{+}$then there exist complementary $T$-invariant bands $H$ and $K$ in $E$ such that $\left.T\right|_{H}$ is central and $T_{K}$ is quasi-nilpotent.

Proof. Once we show that in the decomposition of Theorem 3 each $\left.T\right|_{H_{r}}$ is central, then we can take $H$ to be the closed ideal generated by the $H_{r},\left.T\right|_{H}$ will be central as if $h \in H_{r},|T h| \leqslant\left\|\left.T\right|_{H_{r}}\right\| \cdot|h| \leqslant\|T\| \cdot|h|$, so $\left.T\right|_{\oplus H_{r}}$ is central. The continuity of $T$ will then guarantee that $\left.T\right|_{H}$ is also central.

Theorem 2.1 of [6] in fact tells us that $\left.T\right|_{H_{r}}$ is simply $r$ times the identity on $H$, so is certainly central.

For this result to be true neither the condition that $T^{*}$ be a lattice homomorphism nor that $E$ have an order continuous norm may be dropped.

EXAmple (4). If $T(x, y)=(x, x)$ on $\mathbf{C}^{2}$ then the desired decomposition is not possible.

EXAmpLe (5). Define on $c$ a lattice-homomorphism $T$ by

$$
\begin{aligned}
(T x)_{n} & =\left(x_{n} / n\right) \quad(n \text { even }) \\
& =\left(x_{n+2} / n^{n}\right) \quad(n \text { odd }) .
\end{aligned}
$$

Then $T$ is compact and $T^{*}$ is a lattice homomorphism. If $I=\left\{\left(x_{n}\right) \in c: x_{2 k}=0\right.$ for all $k \in \mathbf{N}\}$, then $\left.T\right|_{I}$ is readily seen to be quasi-nilpotent. We may identify $c / I$ with $c$ and then $T$ takes $\left(y_{n}\right)$ to $\left(y_{n} / 2 n\right)$. Thus $\sigma\left(T_{I}\right)=\{1 / 2 n: n \in \mathbf{N}\} \cup\{0\}$. Since each $1 / 2 n$ clearly lies in $\sigma(T)$, we have that $\sigma(T)=\{1 / 2 n: n \in \mathbf{N}\} \cup\{0\}$ (using the fact that $\left.\sigma(T) \subset \sigma\left(T_{I}\right) \cup \sigma\left(\left.T\right|_{I}\right)\right)$. Thus $T$ has nonnegative spectrum. Any decomposition $c=H \oplus K$ will have one of $H$ or $K$ finite dimensional. If $K$ were finite dimensional then $\left.T\right|_{H}$ would not be central, whilst if $H$ were finite dimensional then $\left.T\right|_{K}$ would not be quasi-nilpotent.

It should be mentioned that the author knows of no example to show that the compactness of $T$ is needed for the result of Corollary 4 to hold. The proof given here of course uses it heavily.

\section{REFERENCES}

1. W. Arendt, Spectral properties of Lamperti operators (preprint).

2. H. P. Lotz, Über das Spektrum positiver Operatoren, Math. Z. 108 (1968), 15-32.

3. Extensions and liftings of positive linear operators on Banach lattices, Trans. Amer. Math. Soc. 211 (1975), 85-100.

4. B. de Pagter, Compact Riesz homomorphisms (preprint).

5. H. H. Schaefer, Banach lattices and positive operators, Springer-Verlag, Berlin, Heidelberg and New York, 1974. 
6. H. H. Schaefer, M. Wolff and W. Arendt, On lattice isomorphisms with positive real spectrum and groups of positive operators, Math. Z. 164 (1978), 115-123.

7. E. Scheffold, Das Spektrum von Verbandsoperatoren in Banachverbänden, Math. Z. 123 (1971), 177-190.

8. B. Walsh, On characterising Köthe sequence spaces as vector lattices, Math. Ann. 175 (1968), $253-256$.

9. A. W. Wickstead, Compact subsets of partially ordered Banach spaces, Math. Ann. 212 (1975), 271-284.

10.

11. Isolated points of the approximate point spectrum of certain lattice homomorphisms on $C_{0}(X)$, Quaestiones Math. 3 (1979), 249-279.

12. __ Extremal structure of cones of operators, Quart. J. Math. Oxford Ser. 232 (1981), 239-253.

13. M. Wolff, Über das Spektrum von Verbandshomomorphismen in $C(X)$, Math. Ann. 182 (1969), 161-169.

14. __ Über das Spektrum von Verbandshomomorphismen in Banach Verbänden, Math. Ann. 184 (1969), 49-55.

Department of PUre Mathematics, QueEn's University, Belfast BT7 INN, Northern Ireland 